the day. Which results make heart failure less likely?

(a) A normal cardiac silhouette on chest radiograph

(b) A normal 12-lead ECG

(c) Normal peak flow and spirometry

(d) A low plasma B-type natriuretic peptide (BNP) concentration

(e) Anterior $Q$ waves on the 12-lead ECG

10 A 55-year-old man with a history of chronic obstructive pulmonary disease presents with increasing breathlessness on exertion and orthopnoea. A transthoracic echocardiogram fails to provide any useful images. What additional tests may help confirm the clinical suspicion of heart failure?

(a) A plasma BNP concentration

(b) Peak flow

(c) A 12-lead ECG

(d) Radionuclide imaging

(e) Full blood count
Guidelines on completing the answer sheet for those who wish to submit their answers on paper

A loose leaf answer sheet is enclosed, which will be marked electronically at the Royal College of Physicians. Answer sheets must be returned by 21 March 2004 to: CME Department (SAQs), Royal College of Physicians, 11 St Andrews Place, London NW1 4LE.

Overseas members only can fax their answers to 02074874156

Correct answers will be published in the next issue of Clinical Medicine.

${ }^{*}$ Further details on CME are available from the CME department at the Royal College of Physicians (address above or telephone 02079351174 extension 306 or 309).

Your completed answer sheet will be scanned to enable a quick and accurate analysis of results. To aid this process, please keep the following in mind:

1 Please print your GMC Number firmly and neatly

2 Only write in allocated areas on the form

3 Only use pens with black or dark blue ink

4 For optimum accuracy, ensure printed numbers avoid contact with box edges

5 Please shade circles like this: Not like this: $\boldsymbol{\sigma}$

6 Please mark any mistakes made like this: $\$$

7 Please do not mark any of the black squares on the corners of each page

8 Please fill in your full name and address on the back of the answer sheet in the space provided; this will be used to mail the form back to you after marking.

\title{
CME Renal Medicine SAQs
}

\section{Answers to the CME SAQs published in Clinical Medicine November/December 2003}

$\begin{array}{llllllllll}\text { Q1 } & \text { Q2 } & \text { Q3 } & \text { Q4 } & \text { Q5 } & \text { Q6 } & \text { Q7 } & \text { Q8 } & \text { Q9 } & \text { Q10 } \\ \text { a) } F & \text { a) } T & \text { a) } F & \text { a) } F & \text { a) } F & \text { a) } F & \text { a) } T & \text { a) } F & \text { a) } T & \text { a) } F \\ \text { b) } F & \text { b) } F & \text { b) } T & \text { b) } F & \text { b) } T & \text { b) } F & \text { b) } F & \text { b) } F & \text { b) } F & \text { b) } T \\ \text { c) } F & \text { c) } F & \text { c) } F & \text { c) } F & \text { c) } F & \text { c) } T & \text { c) } T & \text { c) } T & \text { c) } T & \text { c) } F \\ \text { d) } F & \text { d) } F & \text { d) } F & \text { d) } F & \text { d) } F & \text { d) } F & \text { d) } F & \text { d) } F & \text { d) } T & \text { d) } F \\ \text { e) } T & \text { e) } T & \text { e) } T & \text { e) } T & \text { e) } F & \text { e) } T & \text { e) } F & \text { e) } F & \text { e) } F & \text { e) } F\end{array}$

